Relations industrielles

Industrial Relations

\title{
Mentir au travail, Par Duarte Rolo (2015) Paris : PUF, 142 pages.
}

ISBN : 978-2-13-063531-4.

\section{Frederick Plamondon}

Volume 71, numéro 4, automne 2016

URI : https://id.erudit.org/iderudit/1038531ar

DOI : https://doi.org/10.7202/1038531ar

Aller au sommaire du numéro

Éditeur(s)

Département des relations industrielles de l’Université Laval

ISSN

0034-379X (imprimé)

1703-8138 (numérique)

Découvrir la revue

Citer ce compte rendu

Plamondon, F. (2016). Compte rendu de [Mentir au travail, Par Duarte Rolo (2015) Paris : PUF, 142 pages. ISBN : 978-2-13-063531-4.] Relations industrielles / Industrial Relations, 71(4), 768-769. https://doi.org/10.7202/1038531ar

Tous droits réservés @ C Département des relations industrielles de l’Université Laval, 2016
Ce document est protégé par la loi sur le droit d'auteur. L’utilisation des services d'Érudit (y compris la reproduction) est assujettie à sa politique d'utilisation que vous pouvez consulter en ligne.

https://apropos.erudit.org/fr/usagers/politique-dutilisation/ 


\section{Recensions / Book Reviews}

\section{Mentir au travail}

Par Duarte Rolo (2015) Paris: PUF, 142 pages. ISBN : 978-2-13-063531-4.

La portée de l'ouvrage de Rolo est assez simple à mesurer: il s'agit d'analyser les impacts délétères que causent les dissonances cognitives au sein d'organisations comme les centres d'appels téléphoniques (CAT), soit celles ayant adopté des pratiques de gestion des ressources humaines (GRH) indexant la reconnaissance à la performance individuelle. Ces stratégies, que Rolo associe au néo-taylorisme, visent à persuader les employés de subordonner leur idéal professionnel aux objectifs productivistes de l'employeur, quitte à poser des gestes qu'ils jugent contraires à l'éthique, ce qui pourrait les exposer à des dilemmes éthiques importants et potentiellement pathogènes. À ce titre, I'analyse de Rolo ne déçoit pas: Mentir au travail met en lumière les formes que prennent les relations de pouvoir au travail, ainsi que les impacts de celles-ci sur nos sociétés. Bien que l'influence sociopolitique du néo-taylorisme ne soit pas l'objet principal de Mentir au travail, on ne peut nier que l'ouvrage a été rédigé sur un filigrane politique, véritable clé de lecture dont il faut tenir compte au moment de parcourir ses pages. J'y consacrerai donc les premières lignes de cette recension, pour ensuite commenter la forme et le fond.

D'entrée de jeu, Duarte Rolo reprend des constats qui ne font plus controverse: les profondes transformations que subissent les économies modernes sont en partie responsables de la dégradation du vivre-ensemble, ce qui amène de l'eau au moulin du cynisme politique conduisant à la désaffection des institutions, ainsi qu'à l'apathie des mouvements sociaux. On a qu'à penser à l'échec des Occupy et autres printemps, par exemple. Bref, si la dernière crise économique a appris quelque chose à l'auteur de Mentir au travail, c'est qu'on arrive de moins en moins à envisager des actions collectives subversives, encore moins à mobiliser le peuple, qui semble préférer la "servitude volontaire» à la liberté et à l'autonomie. En somme, ce que Rolo appelle le «tournant gestionnaire » amènerait la pensée, ainsi que la volonté, à capituler devant la force d'un productivisme décomplexé.

Pour dépasser cet état de fait, il propose de regarder du côté du rapport subjectif au travail, notamment en examinant la nature et les fonctions de la souffrance éthique causée par l'adoption de stratégies jugées contradictoires à l'éthique professionnelle. Ainsi, I'organisation néo-taylorienne du travail forcerait les travailleuses et travailleurs à affronter un dilemme: choisir de faire taire leurs consciences et consentir à la démission de leur esprit critique, ou revendiquer une autonomie critique leur permettant de s'affranchir de leur état de servitude volontaire, au risque de ne pas atteindre les objectifs fixés par l'employeur. En apparences, la décision semble tout indiquée (on ne peut pas être contre la vertu après tout), mais il s'agit en fait d'un choix impossible. En effet, peu importe celui-ci, les conséquences ne peuvent être que tragiques. D'une part, si l'employé choisit de faire taire sa conscience, il consent à soumettre l'évaluation de son professionnalisme à des critères hétéronomes (évaluation comparative ou benchmarking en anglais, évaluation quantitative du rendement, qualité totale, etc.) I'incitant à poser des gestes incompatibles avec son idéal professionnel, qui cesse alors de lui appartenir. En somme, la démission de la pensée mène l'employé sur la voie du conformisme et de la dépersonnalisation (ou de la «fonctionnarisation»). D'autre part, s'il choisit l'esprit critique et l'autonomie professionnelle, il risque l'isolement, renonce à la reconnaissance et s'expose à l'opprobre. Par ailleurs, comme ce dilemme est subjectif, il contribue à accentuer l'individualisation du travail, ce qui rend plus difficile la mobilisation des employés et leur 
participation dans l'organisation collective des relations de travail. Finalement, ce dilemme déresponsabilise aussi l'employeur, car peu importe, au fond, le choix que fera l'employé, la direction de l'organisation pourra toujours plaider qu'il s'agissait d'un choix autonome, fait par un professionnel. Bref, I'organisation néo-taylorienne du travail plonge souvent les travailleurs et les travailleuses dans un guet-apens duquel il peut être difficile de s'extraire et qui compromet leur santé psychologique de manière importante.

Sur le fond, l'examen du dilemme éthique et de ses conséquences qu'on retrouve dans Mentir au travail doit son originalité au cadre d'analyse qu'utilise son auteur, qui s'inscrit dans le domaine de la psychodynamique du travail (PDT). La PDT suppose un rapport conflictuel entre l'organisation du travail et l'identité de l'employé. Ce conflit s'exprime à travers un rapport de pouvoir par lequel l'employeur cherche à mettre I'intelligence de l'employé au service de sa fonction première, soit la production d'un rendement. II s'agit évidemment du zèle, qui permet de combler l'écart entre le travail prescrit et le travail réel. De son côté, l'employé souhaite plutôt mettre son intelligence au service de ce qu'il perçoit comme étant la vie bonne. En ce sens, son idéal professionnel joue le rôle d'un idéal régulateur qu'il cherche à réaliser. Pour la PDT, la tension entre les désirs de l'employeur et de l'employé force ce dernier à faire converger son idéal professionnel avec les besoins de l'employeur. En termes psychodynamiques, et dans le meilleur des cas, l'employé peut sublimer cette tension afin de pouvoir réguler son comportement d'une manière qui soit compatible avec son idéal professionnel. À l'inverse, l'échec devient une source de souffrance éthique, ce qui s'incarne à travers le développement de mécanismes de défense permettant de l'endurer. Des mécanismes de défenses, il faut le dire, qui prennent souvent la forme d'une démission de la pensée, d'un appétit pour le conformisme ou d'autres comportements que d'aucuns, par exemple, ont caractérisés comme étant de la «stupidité fonctionnelle» (Alvesson, Spicer : 2012).

Certes, cela rapproche souvent le propos de Mentir au travail de considérations plus philosophiques que ce à quoi on a I'habitude dans les recherches portant sur la santé au travail. Cependant, c'est un aspect du livre de Rolo que j'ai accueilli avec beaucoup d'intérêt, sans doute parce que ma formation est d'abord en philosophie, mais surtout parce qu'il s'agit d'une contribution très féconde, qui ouvre la voie à de nouveaux partenariats entre la santé psychologique au travail et la philosophie. En effet, si on accepte l'existence d'un rapport nécessaire entre l'organisation du travail et la santé psychologique, ce serait une erreur d'écarter les outils capables de rendre compte de l'intimité de la pensée, comme la PDT et la philosophie. À ce sujet, Mentir au travail rappelle que cette dernière pourrait être mise à contribution dans l'analyse des rapports de travail, que ce soit dans des domaines comme l'organisation psychique du travail ou l'ergonomie cognitive, par laquelle le sujet serait invité à développer un souci de soi l'incitant à modifier la culture du travail et, pour faire écho à la conclusion de Rolo, à modifier le monde.

\section{Frederick Plamondon}

Doctorat en Relations industrielles Université Laval

\section{Reflexive Labour Law in the World Society}

Ralf Rogowski (2013) London : Edward Elgar Publishing, 335 pages. ISBN : 978-0-85793658-5.

Cet ouvrage remarquable de Ralf Rogowski, professeur de droit du travail à I'université de Warwick (R.-U.), tire d'abord sa pertinence du fait qu'une appréhension contemporaine de ce droit n'est plus complète, voire satisfaisante, sans une compréhension adéquate des influences mondiales auxquelles il est confronté. Ces dernières 